\title{
Pulse amplitude ratio under noninvasive ventilation as a new method in the diagnosis of heart failure in patients with acute exacerbation of chronic obstructive pulmonary disease
}

Khaoula bel haj ali

Fattouma Bourguiba University Hospital

Adel Sekma

Fattouma Bourguiba University Hospital

Ikram Chamtouri

Fattouma Bourguiba University Hospital

Kaouthar Beltaief

Fattouma Bourguiba University Hospital

Mohamed Amine Msolli

Fattouma Bourguiba University Hospital

\section{Zied Mezgar}

Farhat Hached University Hospital

\section{Wahid Bouida}

Fattouma Bourguiba University Hospital

\section{Riadh Boukef}

Sahloul University Hospital

\section{Hamdi Boubaker}

Fattouma Bourguiba University Hospital

\section{Mohamed Habib Grissa}

Fattouma Bourguiba University Hospital

Semir Nouira ( $\sim$ semir.nouira.urg@gmail.com )

Fattouma Bourguiba University Hospital

\section{Research Article}

Keywords: non-invasive ventilation, chronic obstructive pulmonary disease, heart failure, pulse amplitude ratio

Posted Date: February 24th, 2022 
DOI: https://doi.org/10.21203/rs.3.rs-1374416/v1

License: (c) (1) This work is licensed under a Creative Commons Attribution 4.0 International License. Read Full License 


\section{Abstract}

Background: Heart failure (HF) is commonly associated with acute exacerbation of chronic obstructive pulmonary disease (AECOPD) but its role is often underestimated.

Aim of study: To evaluate the performance of a new diagnostic technique based on the measurement of the pulse amplitude ratio (PAR) using non-invasive ventilation (NIV) for the early identification HF in patients admitted to the emergency department (ED) for AECOPD.

Methods: This is a prospective observational study including patients presenting to the ED with AECOPD. Each included patient received NIV with inspiratory pressure support (PS) set at 5 and $30 \mathrm{~cm} \mathrm{H}_{2} \mathrm{O}$ for 15 min in random order. We measured plethesmographic pulse pressure (PP) at PS of $5 \mathrm{cmH}_{2} \mathrm{O}$ (PP5) and 30 $\mathrm{cmH}_{2} \mathrm{O}$ (PP30). We calculated the PAR under NIV (PAR into two groups: a group identified as having HF (HF group) and a group without HF (non HF group). The diagnostic performance of PAR $\mathrm{NIV}_{\mathrm{V}}$ was assessed by standard methods.

Results: 73 patients were included in this study: 32 in HF group and 41 in non HF- group. The two groups had comparable demographic and clinical characteristics at admission. The mean values of PAR NIV $_{\text {Was }}$ significantly higher among HF patients $(0.86$ versus $0.71 ; p<0.01)$. The area under the receiver operating characteristic curve of PAR ${ }_{\text {NIV }}$ was 0.75 . Using the best cut-off $(0.6)$, the sensitivity of PAR NIV $_{\text {was }} 93 \%$ with a specificity $21 \%$, a positive predictive value of $48 \%$, and a negative predictive value of $81 \%$. Correlation between $\mathrm{PAR}_{\mathrm{NIV}}$ and BNP was significant $(r=0.52 ; \mathrm{p}=0.002)$

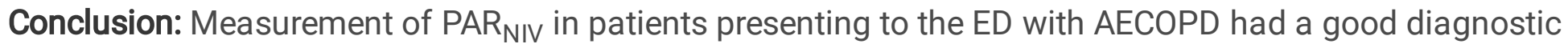
performance for the detection of $\mathrm{HF}$ and could represent an interesting alternative for the currently available methods.

The study was registered in the Clinical Trial Registration System (clinicaltrials.gov) under the study number NCT05189119.

\section{Introduction}

Chronic obstructive pulmonary disease (COPD) is a universally prevalent chronic disease. It is a major public health problem due to its increasing prevalence, high morbidity and mortality rates (1). On average, a COPD patient suffers one to four exacerbations per year. The origin of these exacerbations is not easy to determine, but most often a viral or bacterial infection is incriminated. HF is commonly associated with acute exacerbations of COPD (AECOPD), but this association is frequently underestimated (2-5).

Symptoms and signs of HF are particularly difficult to identify and interpret, especially in obese and elderly subjects $(4,6-9)$. Therefore, the use of additional investigation methods, such as echocardiography and invasive hemodynamic exploration, is decisive for the diagnosis, but they cannot be considered in the emergency room on a routine basis (10). The tendency to generalize cardiac 
ultrasound is still limited by its dependency on the operator skills and experience $(11,12)$. Brain Natriuretic Peptide (BNP) is now widely used for the diagnosis of HF (13). However, a BNP value in the grey zone does not allow the determination of the etiology of dyspnea; this is the case for approximately $30 \%$ of patients consulting the emergency room for dyspnea (14). In addition, elevated BNP levels are observed in the elderly, in patients with renal failure, chronic pulmonary disease and hypoxemia (15). Alternative diagnostic method has been proposed, based on the effect of Valsalva maneuver (VM) on blood pressure (16). VM consists of forced exhalation with a closed glottis, which leads to an increase in intra thoracic pressures, the effect of which on the blood pressure curve depends on whether there is or not HF. Several studies have shown that this maneuver could identify patients with HF through the measurement of the PAR (17-19). Recently, Boubaker et al. found that VM could be used to identify HF in patients with AECOPD (20). However, VM is sometimes not easy to be correctly performed for patients who have difficulties to breath. Thus, we propose, in this study, an alternative method to VM. It consists in applying a positive pressure through NIV with inspiratory support. We aimed to evaluate the value of a new method of measuring PAR based on the application of pressure support ventilation (PSV) to assist in the early diagnosis of $\mathrm{HF}$ in patients presenting to the ED with AECOPD.

\section{Patients And Methods}

This is a prospective observational study of patients who presented to the ED of Fattouma Bourguiba University Hospital (Tunisia) with acute dyspnea in the context of AECOPD. The study protocol has been prepared in accordance with the revised Helsinki Declaration for Biomedical Research Involving Human Subjects and Guidelines for Good Clinical Practice. The study was approved by the ethics committee of our institution; the patient's informed consent was obtained before the start of the protocol. The study was registered in the Clinical Trial Registration System (clinicaltrials.gov) under the study number NCT05189119. This study is reported according to the "Strengthening the Reporting of Observational Studies in Epidemiology (STROBE)" statement guidelines for observational cohort studies.

\section{Study population}

\section{Inclusion criteria}

Adult patients with a history of COPD who consulted the emergency department for moderate to severe AECOPD defined as acute worsening of respiratory symptoms that result in additional therapy were included (2). COPD is defined according to the criteria of the American Thoracic Society as a progressive airflow limitation that is not fully reversible associated with a chronic inflammatory response of the lungs to noxious particles or gases (21).

\section{Exclusion criteria}

Patients with hemodynamic instability (cardiogenic shock, myocardial infarction), coma and severe hypoxemia or acidosis $(\mathrm{pH}<7.1)$ were excluded. Patients with contraindications to NIV (Glasgow score < 12, swallowing disorder, severe bronchial obstruction, massive retention of secretions despite 
bronchoscopy, vomiting, those with anatomical or functional upper airway obstruction or ongoing upper gastrointestinal bleeding or ileus), non-cooperative patients as well as those who refused to give consent were also excluded.

\section{Methodology}

Patients' demographic characteristics, medical histories, number of COPD exacerbations during the last 12 months, smoking and current drug treatment, as well as clinical examination data including blood pressure, heart rate, respiratory rate, pulse oxygen saturation, and temperature were collected.

Complementary examination findings were also recorded (electrocardiogram, chest X-ray, cardiac ultrasound, BNP, blood gas, and standard biological tests). Patients were prepared for monitoring with three-lead electrode ECG and a plethysmographic sensor on the finger to obtain two simultaneous records. The plethysmographic PAR was calculated using a data acquisition and analysis device (BIOPAC Systems, CA, USA). Each enrolled patient received NIV applied through an oro-nasal mask with a positive end expiratory pressure of $4 \mathrm{cmH}_{2} \mathrm{O}$, inspiratory oxygen fraction ensuring pulse oxygen saturation $\geq 94 \%$, and inspiratory pressure support (PS) set at 5 and $30 \mathrm{cmH}_{2} \mathrm{O}$. The 2 PS levels were investigated for 15 min in random order, with 10 min spontaneous breathing in between the ventilation periods to return to baseline level. Three successive measurements of PAR during inspiration time were taken 5 minutes after each level of pressure support onset. The following calculations were performed: PP5 which is the average of the plethysmographic pulse pressure measured during three inspiratory cycles recorded under $\mathrm{PS}=5 \mathrm{cmH}_{2} \mathrm{O}$. PP30 which is the average of the plethysmographic pulse pressure measured during three inspiratory cycles recorded under $\mathrm{PS}=30 \mathrm{cmH}_{2} \mathrm{O}$ (Fig. 1). We defined PAR $\mathrm{NIV}_{\text {as }}$ as the PP30/PP5 ratio. All the measurements were performed by the same investigators who were not aware of the clinical and biological details of the patient. The treating physicians were blinded to the results. The patients were divided into two groups: a group of patients having HF (HF group) and a group of patients without HF (non HF group). The diagnosis of HF was made by two experts on the basis of clinical data, cardiac ultrasound findings and BNP values (22). In case of a disagreement, a third senior physician was consulted and adjudicated the case.

\section{Statistical analysis}

Qualitative data were expressed with frequencies and percentage. Chi-square test or Fisher exact test were used to compare qualitative variables as appropriate. Normality was assessed with the Shapiro-Wilk test for continuous variables. Data are presented as mean (standard deviation) or median (interquartile range) for continuous variables according to their distribution. Comparison of data between the groups was performed using a two-sample Student's t-test or non-parametric tests, as appropriate. We calculated the sensitivity, specificity, negative predictive value and positive predictive value of $P_{A} R_{\text {NIV }}$ using the best cut-off. The choice of the PAR threshold was based on the level having the best sensitivity and specificity on the ROC curve (Youden index). A p-value less than 0.05 was statistically significant. The correlation

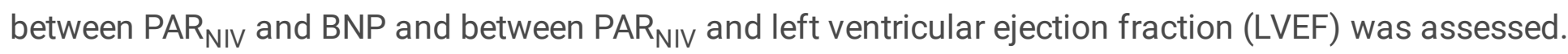
All data were analyzed using the SPSS version 20.0 statistical software. 


\section{Results}

Over the 2-month study period extending from November 2021 to December 2021, 73 patients were included, among whom 32 had a confirmed diagnosis of HF. Table 1 summarizes the characteristics of the overall population. The mean age was $67 \pm 9$ years for the HF group and $68 \pm 10$ years for the non HF group. A male predominance was observed in both groups, with 26 male patients (81\%) in the HF group and 36 patients (87\%) in the non HF group. Previous history of hypertension was found in $46 \%$ of patients and coronary artery disease in $25 \%$ in the HF group. This proportion represented $34 \%$ and $10 \%$, in the non HF group with no statistically significant difference. Mean BNP values were $195 \pm 190 \mathrm{pg} / \mathrm{ml}$ in the non HF group patients and $3421 \pm 1200 \mathrm{pg} / \mathrm{ml}$ in the HF group $(p<0.001)$. LVEF was $43.9 \pm 11 \%$ and $60 \pm 13.5$ in the HF and non HF groups respectively $(p<0.001)$. Mean PAR values were $0.86 \pm 0.12$ in the $\mathrm{HF}$ group and $0.71 \pm 0.14$ in the non HF group $(\mathrm{p}<0.01)$ (Fig. 2). The area under the ROC curve for PAR $\mathrm{R}_{\text {NIV }}$ was 0.75 with the best threshold at $P R_{\text {NIV }}=0.6$ (Fig. 3). Using this cut-off, the sensitivity was $93 \%$, specificity $21 \%$, positive predictive value $48 \%$, and negative predictive value $81 \%$. The correlation between PAR $_{\text {NIV }}$ and BNP was significant $(r=0.52 ; p=0.002)(F i g .4)$. PAR NIV $_{\text {was }}$ inversely correlated with LVEF $(r$ $=-0.337 ; p=0.005)$ (Fig. 5). 
Table 1

Patients' baseline characteristics

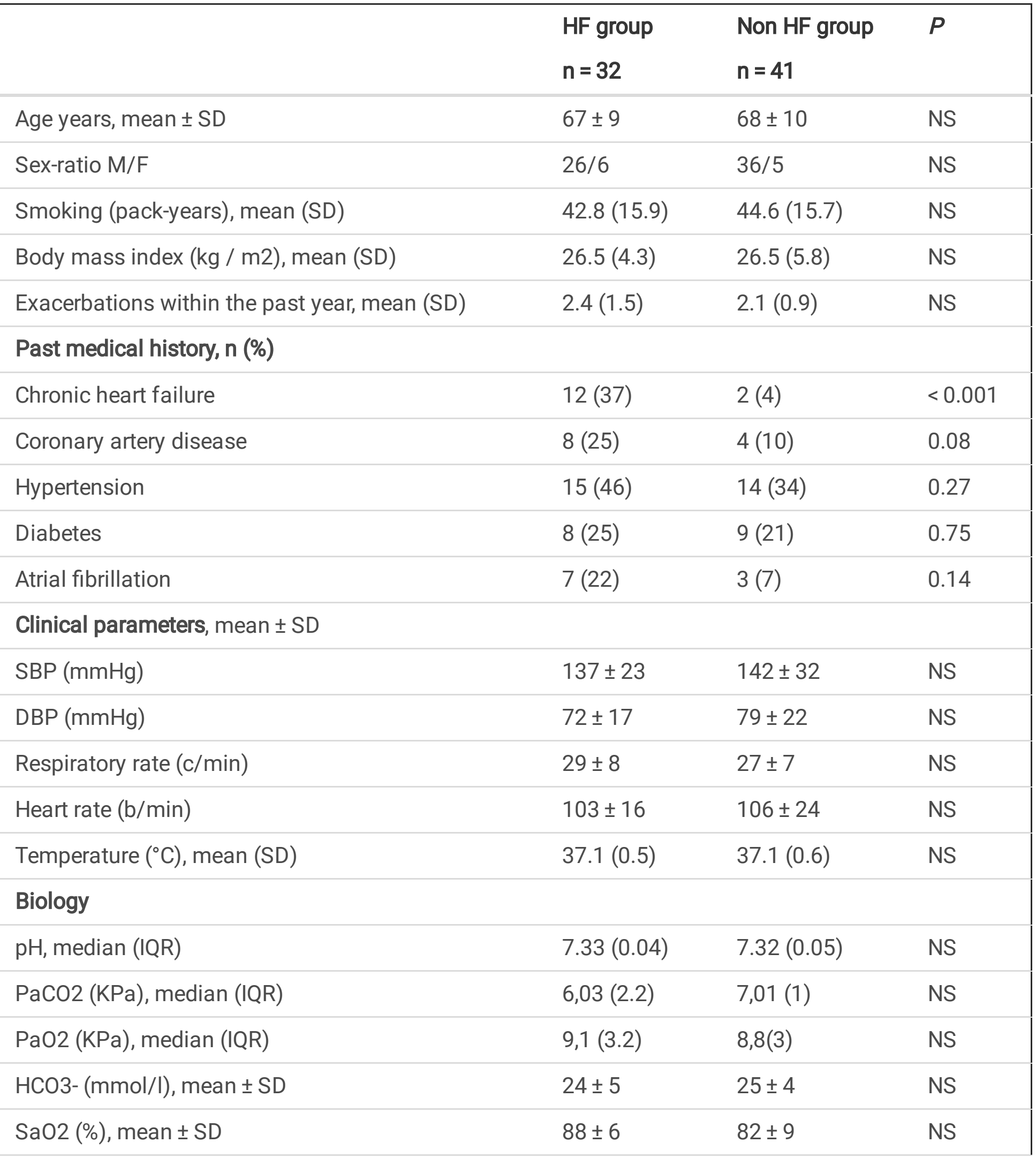

Abbreviations: LVEF: Left Ventricular Ejection Fraction, M/F: male/female, SD: standard deviation, IQR: interquartile range, SBP: Systolic Blood Pressure, DBP: Diastolic Blood Pressure, PaCO2: partial pressure of carbon dioxide, $\mathrm{PaO} 2$ : partial pressure of oxygen, $\mathrm{HCO} 3-$, Serum bicarbonate level, $\mathrm{SaO} 2$ : arterial oxygen saturation, BNP: Brain natriuretic peptide. 


\begin{tabular}{|llll|}
\hline & $\begin{array}{l}\text { HF group } \\
\mathbf{n}=\mathbf{3 2}\end{array}$ & $\begin{array}{l}\text { Non HF group } \\
\mathbf{n}=\mathbf{4 1}\end{array}$ & $\boldsymbol{P}$ \\
\hline BNP(pg/ml), mean \pm SD & $3421 \pm 1200$ & $195 \pm 190$ & $<0.001$ \\
\hline LVEF (\%) : mean \pm SD & $43.9 \pm 11$ & $60 \pm 13.5$ & $<0.001$ \\
\hline $\begin{array}{l}\text { Abbreviations: LVEF: Left Ventricular Ejection Fraction, M/F: male/female, SD: standard deviation, } \\
\text { IQR: interquartile range, SBP: Systolic Blood Pressure, DBP: Diastolic Blood Pressure, PaCO2: partial } \\
\text { pressure of carbon dioxide, PaO2: partial pressure of oxygen, HCO3-, Serum bicarbonate level, SaO2: } \\
\text { arterial oxygen saturation, BNP: Brain natriuretic peptide. }\end{array}$ & & \\
\hline
\end{tabular}

\section{Discussion}

In our study, we proposed a new method using NIV as a mean of HF diagnosis during AECOPD. We found

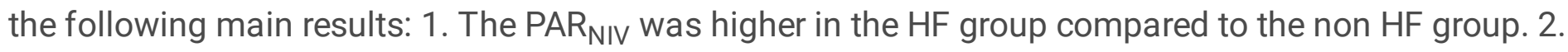

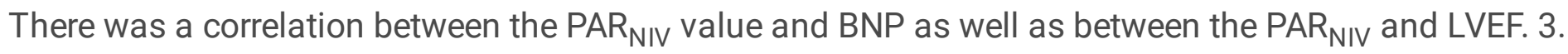

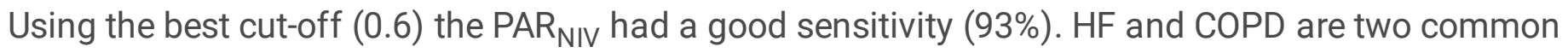
conditions in clinical practice. Both conditions share common risk factors, such as advanced age, smoking, and systemic inflammation which explain their frequent coexistence. Among the co-morbidities most commonly associated with COPD, congestive HF is the most difficult to diagnose leading to a potential detrimental delay in the management of patient's condition, or on the contrary, to unnecessary treatment (6). HF diagnostic uncertainty can be as high as 40\% (23). Chest X-ray and ECG are easily accessible diagnostic tests, but their value in identifying HF is limited in COPD patients $(4,9,24)$. BNP values, as isolated markers of $\mathrm{HF}$, are sometimes insufficient given that a number of non-cardiac conditions may be associated with BNP elevation (13). Diagnosing HF without echocardiography is often challenging and the best diagnostic approach remains unclear. Besides, echocardiography is not always accessible in the ED, and measurements depend on the operator experience. Moreover, the poor acoustic window caused by pulmonary air trapping may hinder accurate echocardiographic assessment of HF in $10-35 \%$ of COPD patients, particularly in those with more severe degrees of airflow obstruction (24). The study of the effect of intra-thoracic pressure variation on the cardiovascular system is likely to provide insight into cardiac function and help in the early and rapid diagnosis of HF. This principle has been applied in numerous studies for the identification of left ventricular dysfunction. One of the most recent studies was conducted by Remmen et al. in which VM and hemodynamic exploration by a Swan-Ganz catheter were performed (25). The results showed a very good correlation between pulmonary capillary wedge (pressure PCWP) and PAR $(r=0.63 ; p<0.001)$. The area under the ROC curve of PAR for the diagnosis of HF (PCWP $>15 \mathrm{mmHg}$ ) was 0.85 . This correlation still remains valid even in patients with arrhythmias. Similar results were found in the study of Wilemann et al. who performed invasive hemodynamic exploration by right catheterization and analyzed the blood pressure response to VM in 42 patients in a stable state (26). They found a very strong correlation between PCWP and PAR $(r=0.75 ; p<$ 0.001). In addition, treatment-induced changes in PCWP correlated well with changes in PAR $(r=0.93 ; p<$ 0.001 . Mclntyre et al. found similar results when they replaced VM with the application of intrathoracic 
positive pressure using airway occlusion under continuous positive airway pressure (27). In mechanically ventilated patients, Zema et al. showed that an abnormal response to VM was associated with a simultaneous increase in central venous pressure and PCWP (28). The rectangular shape of blood pressure record under VM was consistently associated with an increase in PCWP within a range of 20 to $37 \mathrm{mmHg}$. In a recent study, Boubaker et al. showed that plethysmographic PAR during VM was higher in patients with AECOPD with left ventricular dysfunction compared to those without left ventricular dysfunction $(0.78$ vs. $0.38, p=0.01)$. Similarly, they reported that PAR value was not significantly different between COPD group with left ventricular dysfunction and a third group with heart failure and no COPD diagnosis $(p=0.78)$. The area under the ROC curve for plethysmographic PAR to detect left ventricular dysfunction was significantly higher compared to that of the Boston score $(0.92$ vs $0.76 ; p=0.02)(20)$. In practice, VM is difficult to apply in COPD patients in respiratory distress. Therefore, in our study, we proposed the application of NIV using two levels of PS (5 and $\left.30 \mathrm{cmH}_{2} \mathrm{O}\right)$ to simulate the effects of VM. To our knowledge, this is the first study to use this method as a diagnostic tool of HF in AECOPD patients.

Our study has some limitations. First, we used the plethysmographic signal instead of the invasive blood pressure records. It is true that this signal can be modified by artifacts, but it was shown that the respiratory variations of the invasive and non-invasive blood pressure values were correlated (29). Second limitation of the present research that deserves to be highlighted is the choice of the level of PS. This level must be sufficient to create the intrathoracic pressure necessary to induce an effect on the cardiovascular system. We have used a level of $30 \mathrm{cmH} 20$ because beyond this level there is a risk of increasing leakage through the respiratory circuit and abdominal distension. Finally, our technique does not allow to affirm the role of $\mathrm{HF}$ in the actual exacerbation episode of COPD.

In conclusion, the $\mathrm{PAR}_{\text {NIV }}$ measurement could become in the future a useful index to facilitate the diagnosis of HF in AECOPD patients. Our results should be confirmed on a larger sample of patients.

\section{Abbreviations}

HF: Heart failure

COPD: Chronic obstructive pulmonary disease

AECOPD: acute exacerbation of chronic obstructive pulmonary disease

PAR: pulse amplitude ratio

NIV: non-invasive ventilation

ED: emergency department

PS: pressure support

PP: plethesmographic pulse pressure 
AUC: area under roc curve

BNP: Brain Natriuretic Peptide

VM: Valsalva maneuver

PSV: pressure support ventilation

LVEF: left ventricular ejection fraction

PCPW: pulmonary capillary wedge pressure

\section{Declarations}

Ethics approval and consent to participate

The study protocol has been prepared in accordance with the revised Helsinki Declaration for Biomedical Research Involving Human Subjects and Guidelines for Good Clinical Practice. The study was approved by the ethics committee of our institution (Fattouma Bourguiba University hospital ethic committee). For all included patients, written informed consent was obtained before enrollment.

\section{Consent for publication}

Not applicable

\section{Availability of data and materials}

The datasets generated and/or analysed during the current study are not publicly available but are available from the corresponding author on reasonable request.

\section{Competing interests}

The authors declare that they have no competing interests.

\section{Funding}

This research did not receive any specific grant from funding agencies in the public, commercial, or notfor-profit sectors.

\section{Authors' contributions}

SN and RB conceived the study and Owrite the manuscript. SN and RB participated in the design of the study. SN and KBHA revised the manuscript. AS, IC, MAM, ZM and KB collected the data. KB, MHG and $\mathrm{HB}$ participated in the design of the study and performed the statistical analysis. All authors discussed the results and commented on the manuscript. 
The authors acknowledge all of our Research Laboratory members who contributed greatly to this study.

\section{References}

1. Celli BR, Wedzicha JA. Update on Clinical Aspects of Chronic Obstructive Pulmonary Disease. N Engl J Med. 2019;381(13):1257-66.

2. Singh D, Agusti A, Anzueto A, Barnes PJ, Bourbeau J, Celli BR, et al. Global Strategy for the Diagnosis, Management, and Prevention of Chronic Obstructive Lung Disease: the GOLD science committee report 2019. Eur Respir J. 2019;53(5):1900164.

3. Vestbo J, Hurd SS, Agustí AG, Jones PW, Vogelmeier C, Anzueto A, et al. Global strategy for the diagnosis, management, and prevention of chronic obstructive pulmonary disease: GOLD executive summary. Am J Respir Crit Care Med. 2013;187(4):347-65.

4. Hawkins NM, Petrie MC, Jhund PS, Chalmers GW, Dunn FG, McMurray JJV. Heart failure and chronic obstructive pulmonary disease: diagnostic pitfalls and epidemiology. Eur J Heart Fail. 2009;11(2):130-9.

5. Iversen KK, Kjaergaard J, Akkan D, Kober L, Torp-Pedersen C, Hassager C, et al. Chronic obstructive pulmonary disease in patients admitted with heart failure. J Intern Med. 2008;264(4):361-9.

6. Le Jemtel TH, Padeletti M, Jelic S. Diagnostic and therapeutic challenges in patients with coexistent chronic obstructive pulmonary disease and chronic heart failure. J Am Coll Cardiol. 2007;49(2):17180.

7. Ambrosino N, Serradori M. Determining the cause of dyspnoea: linguistic and biological descriptors. Chron Respir Dis. 2006;3(3):117-22.

8. Boonman-de Winter LJM, Rutten FH, Cramer MJ, Landman MJ, Zuithoff NPA, Liem AH, et al. Efficiently screening heart failure in patients with type 2 diabetes. Eur J Heart Fail. 2015;17(2):18795.

9. Rutten FH, Moons KGM, Cramer M-JM, Grobbee DE, Zuithoff NPA, Lammers J-WJ, et al. Recognising heart failure in elderly patients with stable chronic obstructive pulmonary disease in primary care: cross sectional diagnostic study. BMJ. 2005;331(7529):1379.

10. Wang CS, FitzGerald JM, Schulzer M, Mak E, Ayas NT. Does this dyspneic patient in the emergency department have congestive heart failure? JAMA. 2005;294(15):1944-56.

11. Gallard E, Redonnet J-P, Bourcier J-E, Deshaies D, Largeteau N, Amalric J-M, et al. Diagnostic performance of cardiopulmonary ultrasound performed by the emergency physician in the management of acute dyspnea. Am J Emerg Med. 2015;33(3):352-8.

12. Price S, Platz E, Cullen L, Tavazzi G, Christ M, Cowie MR, et al. Expert consensus document: Echocardiography and lung ultrasonography for the assessment and management of acute heart failure. Nat Rev Cardiol. 2017;14(7):427-40. 
13. Nadar SK, Shaikh MM. Biomarkers in Routine Heart Failure Clinical Care. Card Fail Rev. 2019;5(1):50-6.

14. Collins S, Storrow AB, Kirk JD, Pang PS, Diercks DB, Gheorghiade M. Beyond pulmonary edema: diagnostic, risk stratification, and treatment challenges of acute heart failure management in the emergency department. Ann Emerg Med. 2008;51(1):45-57.

15. Maisel A, Mueller C, Adams K, Anker SD, Aspromonte N, Cleland JGF, et al. State of the art: using natriuretic peptide levels in clinical practice. Eur J Heart Fail. 2008;10(9):824-39.

16. Pinsky MR. Applied cardiovascular physiology in theatre: measuring the cardiovascular effects of propofol anaesthesia. Br J Anaesth. 2016;116(6):736-8.

17. Bernardi L, Saviolo R, Spodick DH. Do hemodynamic responses to the valsalva maneuver reflect myocardial dysfunction? Chest. 1989;95(5):986-91.

18. Judson WE, Hatcher JD, Wilkins RW. Blood pressure responses to the Valsalva maneuver in cardiac patients with and without congestive failure. Circulation. 1955;11(6):889-99.

19. Gilotra NA, Wanamaker BL, Rahim H, Kunkel K, Yenokyan G, Schulman SP, et al. Usefulness of Noninvasively Measured Pulse Amplitude Changes During the Valsalva Maneuver to Identify Hospitalized Heart Failure Patients at Risk of 30-Day Heart Failure Events (from the PRESSURE-HF Study). Am J Cardiol. 2020;125(6):916-23.

20. Boubaker H, Grissa MH, Debbabi H, Beltaief K, Dridi Z, Najjar MF, et al. Value of Plethysmographic Pulse Amplitude Ratio With Valsalva Maneuver in Identifying Left Ventricular Dysfunction During COPD Exacerbation. Respir Care. 2016;61(4):439-46.

21. Celli BR, Decramer M, Wedzicha JA, Wilson KC, Agustí A, Criner GJ, et al. An Official American Thoracic Society/European Respiratory Society Statement: Research Questions in Chronic Obstructive Pulmonary Disease. Am J Respir Crit Care Med. 2015;191(7):e4-27.

22. Ponikowski P, Voors AA, Anker SD, Bueno H, Cleland JGF, Coats AJS, et al. 2016 ESC Guidelines for the diagnosis and treatment of acute and chronic heart failure: The Task Force for the diagnosis and treatment of acute and chronic heart failure of the European Society of Cardiology (ESC)Developed with the special contribution of the Heart Failure Association (HFA) of the ESC. Eur Heart J. 2016;37(27):2129-200.

23. Mascarenhas $\mathrm{J}$, Azevedo A, Bettencourt P. Coexisting chronic obstructive pulmonary disease and heart failure: implications for treatment, course and mortality. Curr Opin Pulm Med. 2010;16(2):10611.

24. Boussuges A, Pinet C, Molenat F, Burnet H, Ambrosi P, Badier M, et al. Left atrial and ventricular filling in chronic obstructive pulmonary disease. An echocardiographic and Doppler study. Am J Respir Crit Care Med. 2000;162(2 Pt 1):670-5.

25. J. Remmen, M. Aengevaeren, A. Verheugt, M. Jansen; Detection of elevated pulmonary capillary wedge pressure in elderly patients with various cardiac disorders by the Valsalva manoeuvre. Clin Sci. 2006; 111 (2): 153-162. 
26. Weilenmann D, Rickli H, Follath F, Kiowski W, Brunner-La Rocca HP. Noninvasive evaluation of pulmonary capillary wedge pressure by BP response to the Valsalva maneuver. Chest. 2002;122(1):140-5.

27. McIntyre KM, Vita JA, Lambrew CT, Freeman J, Loscalzo J. A noninvasive method of predicting pulmonary-capillary wedge pressure. N Engl J Med. 1992;327(24):1715-20.

28. Zema MJ. Diagnosing heart failure by the Valsalva maneuver: Isn't It finally time? Chest. 1999;116(4):851-3.

29. Natalini G, Rosano A, Franceschetti ME, Facchetti P, Bernardini A. Variations in arterial blood pressure and photoplethysmography during mechanical ventilation. Anesth Analg. 2006;103(5):1182-8.

\section{Figures}
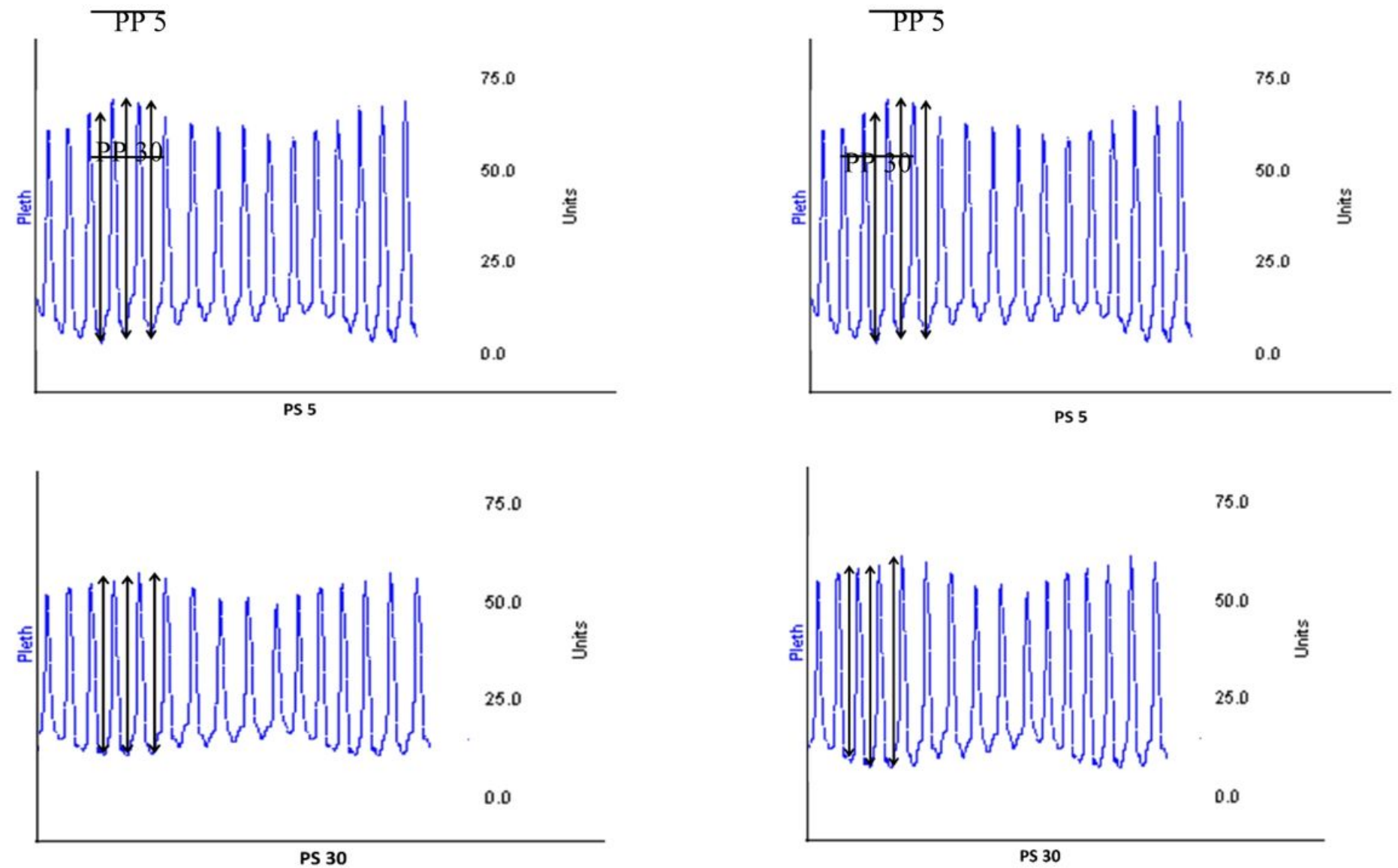

Figure 1

Measurement of Pulse amplitude ratio using plethysmographic blood pressure signal under non-invasive ventilation with two levels of pressure support ( 5 and $30 \mathrm{cmH} 20)$. PP $5=$ average of 3 consecutives pulse pressure values under pressure support of $5 \mathrm{cmH} 2 \mathrm{O}$. PP30 = average of 3 consecutive pulse pressure values under pressure support of $30 \mathrm{cmH} 2 \mathrm{O}$. 
Abbreviations: HF: heart failure.

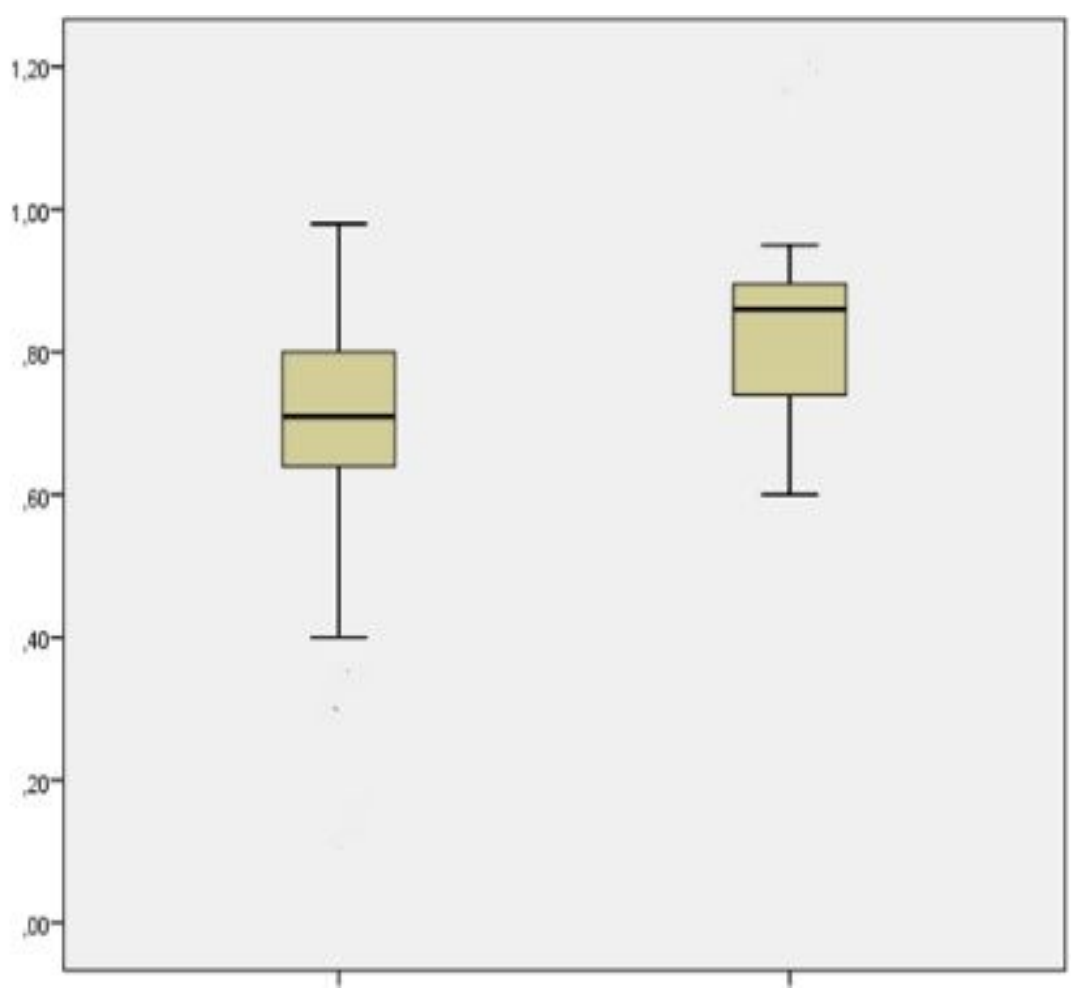

Figure 2

Pulse amplitude ratio under non-invasive ventilation in patients with heart failure (HF group) and patients without heart failure (non HF group)

Abbreviations: PAR $_{\text {NIV }}$ : Pulse amplitude ratio under non-invasive ventilation, HF: heart failure. 


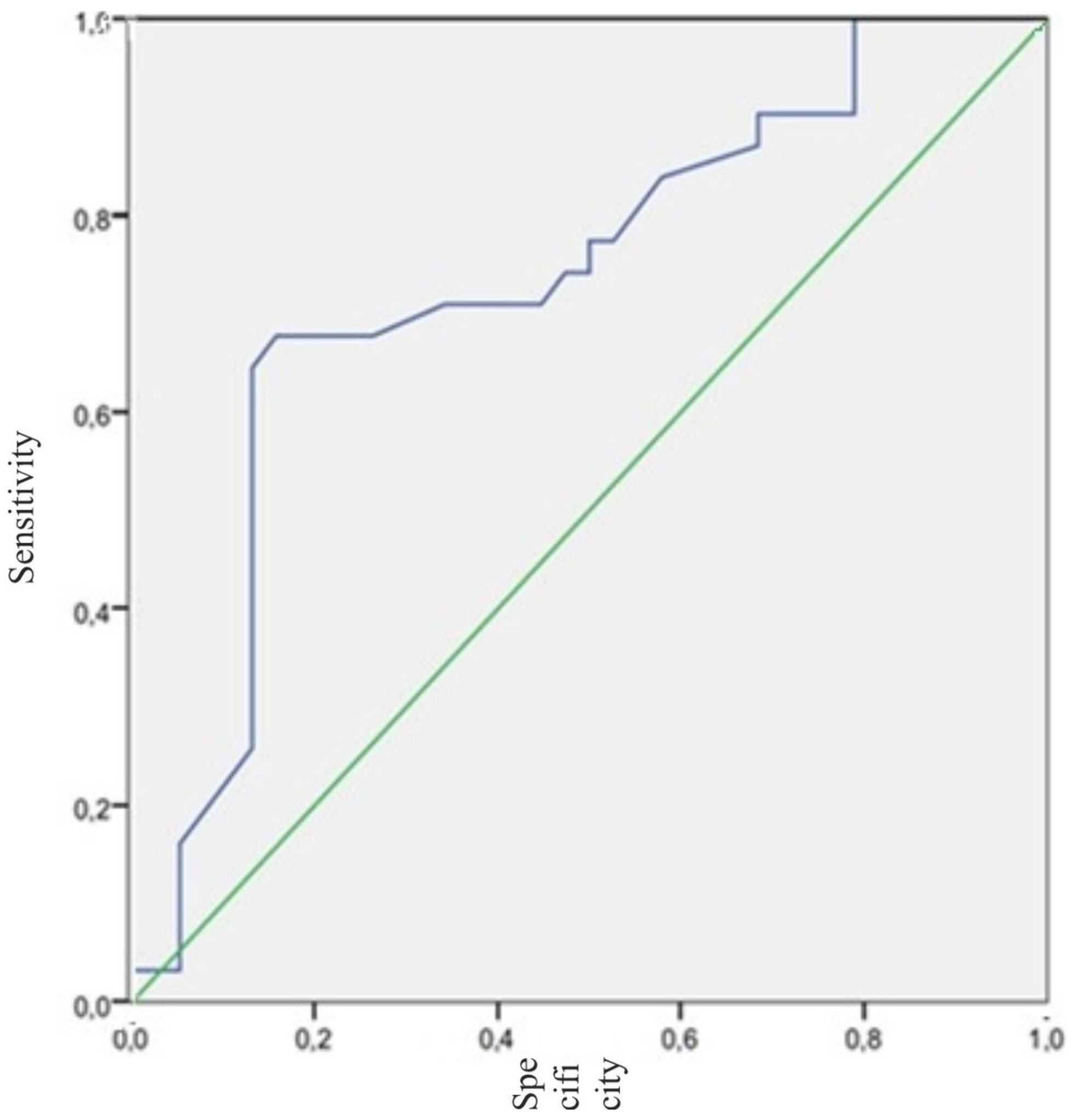

Figure 3

Area under the ROC curve of Pulse amplitude ratio under non-invasive ventilation.

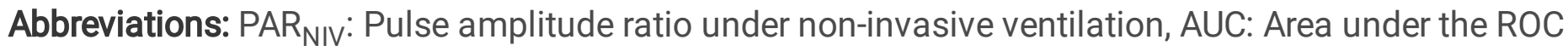
curve. 


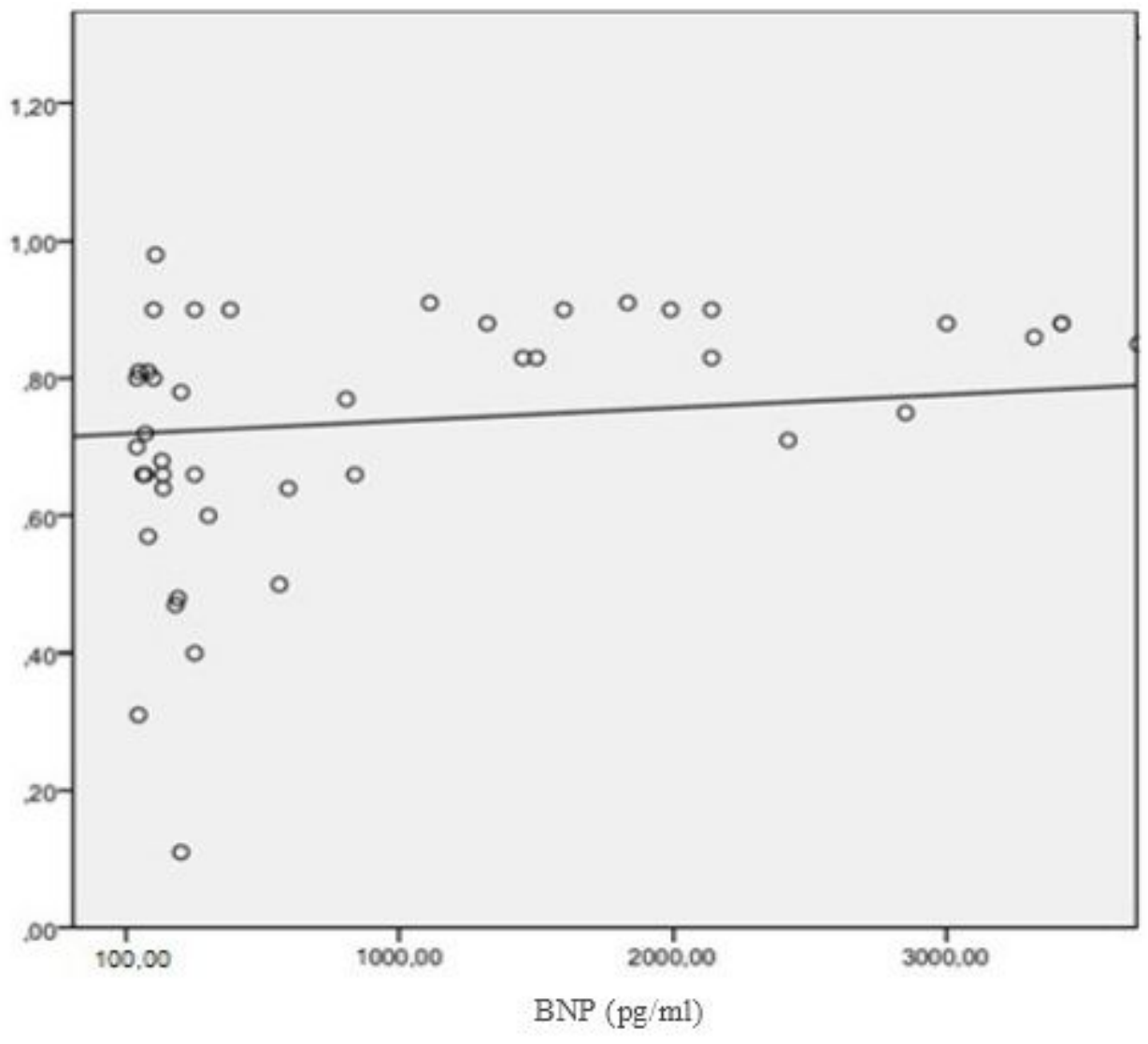

Figure 4

Correlation between Pulse amplitude ratio under non-invasive ventilation and BNP values.

Abbreviations: PAR $_{\text {NIV }}$ : Pulse amplitude ratio under non-invasive ventilation, BNP: brain natriuretic peptide. 


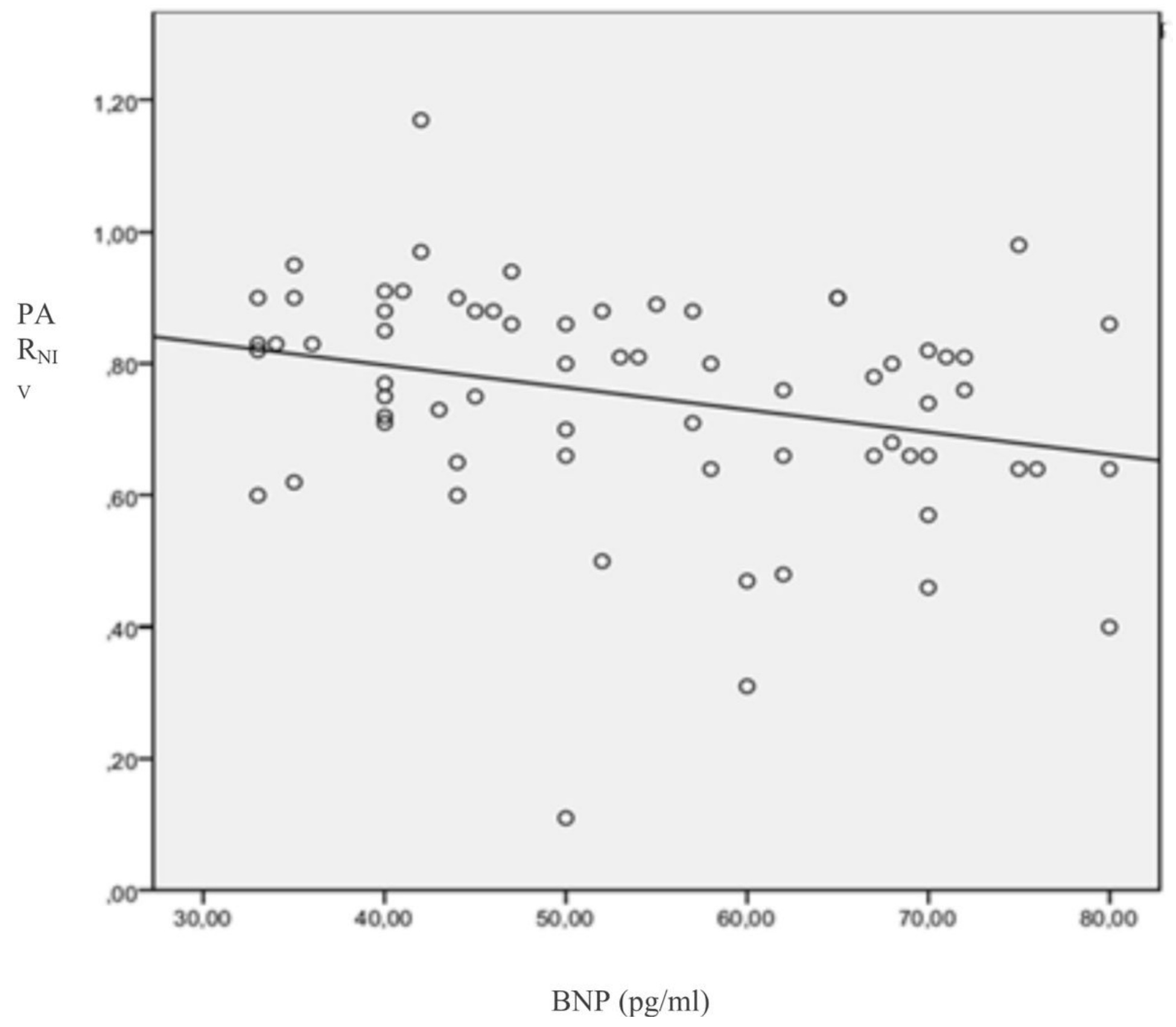

Figure 5

Correlation between Pulse amplitude ratio under non-invasive ventilation and left ventricular ejection fraction values.

Abbreviations: PAR $_{\text {NIV }}$ : Pulse amplitude ratio under non-invasive ventilation, LVEF: left ventricular ejection fraction. 